\title{
ESTRUTURA TERMAL EM ALTA RESOLUÇÃO DAS ÁGUAS DA FRENTE POLAR NA REGIÃO DA PASSAGEM DE DRAKE DURANTE AS OPERAÇÕES ANTÁRTICAS XXII E XXIII (VERÃO AUSTRAL DE 2003 E 2004)
}

\author{
LEILA BAGANHA RABELO ${ }^{1}$, RONALD BUSS DE SOUZA ${ }^{1}$, MAURICIO MAGALHÃES MATA ${ }^{2}$ \& MARCELO FREITAS SANTINI ${ }^{1}$ \\ ${ }^{1}$ Instituto Nacional de Pesquisas Espaciais, Centro Regional Sul de Pesquisas Espaciais, Caixa Postal 5021, Santa Maria, RS, Brasil. CEP: \\ 97105-970, leilaoceano@gmail.com \\ ${ }^{2}$ Universidade Federal do Rio Grande, Instituto de Oceanografia, Av. Itália km 8, RS, Brasil. CEP: 96201-900, mauricio.mata@furg.br
}

\section{RESUMO}

Através do Programa Antártico Brasileiro (PROANTAR), muitos estudos tem se dedicado a vários aspectos da oceanografia antártica e aos processos de tele conexão entre o oceano Austral e o oceano Atlântico Sul. Valendo-se de dados de batitermógrafos descartáveis (XBTs), coletados em alta resolução durante a derrota do Navio de Apoio Oceanográfico (NApOc) Ary Rongel na região da Passagem de Drake, esse trabalho analisa a estrutura termal da Frente Polar (FP) durante o mês de novembro dos anos de 2003 e 2004. A temperatura média da água na superfície do mar foi de $4,1^{\circ} \mathrm{C}$ na Zona Subantártica (ZSA - região norte da FP) e $-0,4^{\circ} \mathrm{C}$ na Zona Antártica (ZA - região sul da FP), valores que indicam o alto contraste térmico superficial entre as massas d'água que se encontram ao longo da FP. Com base na metodologia proposta por Kara et al. (2000), determinou-se através do máximo gradiente horizontal de temperatura que a FP estava situada nas proximidades de $58^{\circ} \mathrm{S}$ no período desse estudo. As características oceanográficas gerais da região de estudo podem ser resumidas dessa forma: (i) em profundidades maiores que $200 \mathrm{~m}$, a ZA apresenta águas com temperaturas inferiores a $2^{\circ} \mathrm{C}$ e cerca de $2,5^{\circ} \mathrm{C}$ mais quentes que as águas superficiais; (ii) em profundidades maiores que $200 \mathrm{~m}$, a ZSA apresenta águas com temperaturas entre $2,5^{\circ} \mathrm{C}$ e $3^{\circ} \mathrm{C}$ e cerca de $1^{\circ} \mathrm{C}$ a $1,5^{\circ} \mathrm{C}$ mais frias que as águas superficiais; (iii) a ZA apresenta uma camada de mistura mais estável até $100 \mathrm{~m}$ de profundidade, onde se encontra o topo da termoclina; (iv) a ZSA apresenta uma termoclina que se estende desde a superfície até a profundidade de $200 \mathrm{~m}$. Estudos descritivos como o apresentado aqui aumentam nosso conhecimento sobre a natureza da FP e sua variabilidade. A modelagem numérica de circulação oceânica pode ser altamente beneficiada pela disponibilidade de novos dados de temperatura da água do mar e da profundidade da termoclina coletados in situ com alta resolução. A determinação precisa da espessura da camada de mistura oceânica na região da Passagem de Drake é importante para auxiliar o entendimento do acoplamento oceano-atmosfera e os processos de teleconexão entre o oceano Austral e o oceano Atlântico Sul.

PALAVRAS CHAVE: Frente Polar; temperatura da água do mar; oceano Austral; batitermógrafo descartável.

\section{ABSTRACT}

High resolution thermal structure of the Polar Front waters at the Drake Passage region during the 22nd and 23rd Brazilian Antarctic expeditions (Austral summer 2003 and 2004).

The Brazilian Antarctic Program (PROANTAR) has fomented several studies regarding to many aspects of the Antarctic oceanography and of the teleconnection processes between the Southern ocean and the South Atlantic. Using Expandable Bathy-Thermographs (XBTs) data collected at high resolution by the Oceanographic Support Ship Ary Rongel in the Drake Passage region, this study analyses the thermal structure of the Polar Front (PF) during the months of November 2003 and 2004. The mean sea surface temperature was $4,1^{\circ} \mathrm{C}$ in the Subantarctic Zone (SAZ - North of the PF) and $-0,4^{\circ} \mathrm{C}$ in Antarctic Zone (AZ - South of the PF), values that indicate the high surface thermal contrast between the water masses meeting along the PF. Based in the methodology proposed by Kara et al. (2000) for computing the maximum temperature horizontal gradient, this work found that the PF was situated close to $58^{\circ} \mathrm{S}$ in the period of this study. The general oceanographic characteristics of the study region can be resumed as follows: (i) at depths deeper than $200 \mathrm{~m}$ the AZ presents water temperatures colder than $2^{\circ} \mathrm{C}$ and about $2,5^{\circ} \mathrm{C}$ warmer than at the surface; (ii) at depths deeper than $200 \mathrm{~m}$ the SAZ presents waters with temperatures ranging from $2,5^{\circ} \mathrm{C}$ to $3^{\circ} \mathrm{C}$ and about $1^{\circ} \mathrm{C}$ to $1,5^{\circ} \mathrm{C}$ colder than at the surface; (iii) the $\mathrm{AZ}$ presents a more stable mixture layer down to the $100 \mathrm{~m}$ depth where the top of the thermocline is located; (iv) the SAZ presents a thermocline spreading itself from the surface to the $200 \mathrm{~m}$ depth. Descriptive studies like the one presented here increase our knowledge on the PF nature and variability. The numeric modeling of the ocean circulation can be highly benefited by the availability of new, high resolution water temperature and thermocline depth data collected in situ. The accurate determination of the ocean's mixing layer width in the Drake Passage region is important for increasing our understanding of the ocean-atmosphere coupling and the teleconnection processes between the Southern and the South Atlantic oceans.

KEYWORDS: Polar Front; Temperature; Southern ocean, Expandable bathythermograph.

\section{INTRODUÇÃO}

O oceano Austral é o único oceano verdadeiramente global do planeta, comunicando-se com os três grandes oceanos (Pacífico, Atlântico e Índico). As regiões limítrofes entre esses oceanos possuem significativa importância devido aos processos físicos que ocorrem na interface de suas águas, realizando transferência de propriedades físicas como calor, sal e momentum. Nas porções próximas ao continente antártico, processos locais como formação e derretimento de gelo marinho, interação entre frentes e correntes oceânicas e a mistura entre massas de águas de diferentes origens fazem com que oceano Austral sirva como fonte de formação de águas de fundo que integram o sistema climático global através da célula de revolvimento meridional. Por essa razão, além de sua importância sob o ponto de vista biológico, o oceano Austral tem sido objeto de interesse de diversos estudos oceanográficos (Dong et al. 2006).

No Brasil, o estabelecimento do Grupo de Oceanografia de Altas Latitudes (GOAL) dentro do 
PROANTAR (Programa Antártico Brasileiro) tem por objetivo fornecer o entendimento de relações entre o ambiente físico/químico, os microorganismos marinhos, e os predadores de topo da cadeia trófica no oceano Austral. Concomitantemente, são realizados pelo PROANTAR estudos dos processos de teleconexão entre o oceano Austral e o ambiente subtropical na porção sul-sudeste do Brasil. Para atender esses objetivos, o GOAL tem efetuado observações meteooceanográficas in situ desde a Operação Antártica XXI (2002) não somente no oceano Austral, mas na porção sudoeste do oceano Atlântico durante a derrota do Navio de Apoio Oceanográfico (NApOc) Ary Rongel entre o Brasil e a Antártica. Estas observações têm sido usadas para estudos avançados de interação oceanoatmosfera (eg. Pezzi et al., 2005, 2009).

Dados observacionais referentes à estrutura térmica da coluna d'água, correntes superficiais dos oceanos e sondagens atmosféricas têm sido obtidos com êxito nos últimos anos através do uso de instrumentos lançados a partir do NApOc Ary Rongel. Ao longo da derrota do navio, três áreas são de interesse direto do GOAL: a região da Confluência Brasil-Malvinas no oceano Atlântico Sudoeste, a região da quebra de plataforma continental da Patagônia Argentina e a Passagem de Drake (Figura 1c).

A Passagem de Drake é a região entre o Cabo Hornos, ao sul da América do Sul e o norte da Península Antártica. Com aproximadamente $700 \mathrm{~km}$ de extensão, a Passagem de Drake afunila o trajeto da Corrente Circumpolar Antártica (CCA), que é a única corrente marinha capaz de dar a volta no globo. A CCA é uma corrente muito profunda e intensa, chegando a transportar até $134 \mathrm{~Sv}\left(134 \times 10^{6} \mathrm{~m}^{3} \mathrm{~s}^{-1}\right.$, Cunninghan et al., 2003). Sua existência é devida a atuação do vento do quadrante oeste, por isso a CCA era também conhecida como "Deriva do Vento de Oeste" (Pickard 1974). Esta região é muito importante para a realização de estudos, pois é o local onde as variabilidades de massa e fluxos de propriedades são mais intensos e podem ser estudados mais facilmente a partir de medidas diretas (Sprintall 2003).

A CCA é composta por três grandes frentes circumpolares, sendo elas: a Frente Subantártica (FSA), a Frente Polar (FP), também conhecida como Convergência Antártica (Dong et al. 2006) e a Frente Circumpolar Antártica Austral (FCAA). As frentes separam massas de água superficiais distintas e são associadas com fortes correntes e fortes gradientes laterais de temperatura, salinidade e produtividade biológica (Moore et al. 1997, Moore et al. 1999, Dong et al. 2006). Cada frente tem extensão circumpolar e estende-se da superfície do mar até o fundo oceânico. Critérios simples baseados na temperatura e salinidade podem ser utilizados para determinar a localização das frentes sobre seções hidrográficas (Sokolov \& Rintoul 2009).

A FCCA pode ser claramente identificada por um decréscimo na salinidade na camada oceânica onde se observa um mínimo de temperatura mínima (Heywood \& King 2002).

Existem diversos estudos que discutem a definição da FP. Por se tratar de uma zona com grande variabilidade tanto nas características físicas das massas d'água e correntes quanto na sua posição geográfica, naturalmente existem diferentes métodos de reconhecimento. Alguns autores optam como definição da posição da FP o limite norte de formação da Água de Fundo Antártica (Cunningham et al. (2003), outros pelo mínimo de temperatura encontrado a 200 m (Deacon, 1937; Belkin e Gordon, 1996) ou até pelo limite da inversão de temperatura em subsuperfície em relação à superfície, entre outras maneiras (Gordon 1971). Whitworth III \& Nowlin (1987) sugerem a identificação da FSA como - local de um segundo mínimo de temperatura direcionado para o norte da Água Intermediaria Antártica. A FP, ao longo de sua distribuição circumpolar, pode ocupar latitudes maiores ou menores em função da topografia de fundo, regime de ventos, fatores termohalinos e/ou sazonalidade. Segundo Brown (1998) e Dong et al. (2006), a FP se posiciona geralmente entre $57^{\circ} \mathrm{S}$ e $60^{\circ} \mathrm{S}$ na região da passagem de Drake. Gordon (1967) aponta que a FP se trata de uma zona com $2^{\circ}$ a $4^{\circ}$ de latitude em largura. Moore et al. (1997), através da análise de imagens de temperatura da superfície do mar (TSM) obtidas com o sensor AVHRR (Advanced Very High Resolution Radiometer), definiram a FP a partir de um gradiente meridional de temperatura superficial de $1,35^{\circ} \mathrm{C}$ por uma distância de 45 a $65 \mathrm{~km}$, variando em função da latitude e longitude em que a frente ocupa.

Park et al. (1998) estudaram a FP na região entre os oceanos Índico e Austral e relataram que a 
natureza altamente meandrante da frente deve-se a atuação de processos de mesoescala. Gouretski e Danilov (1993) observaram que a formação dessas estruturas está relacionada com a batimetria da cordilheira sudoeste Indiana. Glorioso et al. (2005), analisando as características de um vórtice presente no oceano Atlântico Sudoeste, indicaram que esse pode ter sido destacado da região da FP. Sua estrutura vertical de temperatura, com temperatura inferior a $1,5^{\circ} \mathrm{C}$ localizada próxima a $200 \mathrm{~m}$ foi considerada típica de águas antárticas. Estudos como os de Glorioso et al. (2005) atestam para a importância de estudar as características termais das águas da CCA e da FP para o entendimento dos processo de mistura entre os oceanos Atlântico Sul e Austral.

Esse estudo objetiva caracterizar a estrutura termal da região da FP utilizando dados de batitermógrafos descartáveis ou XBTs (Expandable Bathithermographs) em alta resolução espacial $\left(0,25^{\circ}\right.$ de latitude) coletados pelo GOAL nos anos 2003 e 2004 durante as Operações Antárticas XXII e XXIII (OPXXII e OPXXIII, respectivamente). As áreas específicas de análise desse trabalho são a Zona Antártica (ZA - região que vai do continente Antártico até a FP) e a Zona Subantártica (ZSA - região entre a Frente Polar e a Frente Subtropical - Pickard, 1974). A partir de dados obtidos com os XBTs, é realizada uma caracterização das correntes e massas d'água das ZA e ZSA, descrevendo também sua variabilidade entre os anos de 2003 e 2004. O presente trabalho também oferece um método para a determinação da profundidade da termoclina na ZA e na ZSA. Os dados resultantes são importantes para a estimativa da espessura da camada de mistura oceânica na região.

\section{CORRENTES E MASSAS D'ÁGUA}

A CCA apresenta algumas variações quanto à sua direção ao redor do continente Antártico, algumas relacionadas diretamente com a batimetria. Após cruzar a Passagem de Drake, a CCA gera um ramo na direção norte que dá origem à Corrente das Malvinas. Essa última, por sua vez, flui ao longo da região de quebra de plataforma continental da Patagônia Argentina até encontrar a Corrente do Brasil, que flui em sentido contrário. Esse encontro de águas de origem subantártica com águas de origem subtropical forma a Confluência Brasil-Malvinas (CBM, Figura 1.c).
A CBM marca o limite oeste da Frente Subtropical no oceano Atlântico Sul. Apesar de transportar um dos maiores volumes de água oceânica do mundo, a CCA não é muito veloz, quando comparada com outras correntes como a Corrente do Brasil ou mesmo a Corrente das Malvinas. Sua velocidade chega a 4 $\mathrm{cm} . \mathrm{s}^{-1}$ na ZA e $15 \mathrm{~cm} . \mathrm{s}^{-1}$ na ZSA, ambas na camada que se estende desde a superfície até o fundo (Pezzi et al., 2005, 2009).

O método mais comum para a identificação de uma frente oceanográfica é a observação do gradiente de temperatura e salinidade em uma seção perpendicular à frente. Entretanto, esse método pode se tornar errôneo para a identificação da FP, uma vez que pode não existir uma variação tão distinta de temperatura entre a ZA e a ZSA. Essa condição ocorre em duas situações no verão austral: quando o aquecimento das camadas superficiais elimina 0 gradiente ou quando uma fina camada de Água Superficial Subantártica (ASS) flui sobre a Água Superficial Antártica (ASA). Por esta razão, opta-se por utilizar uma característica de subsuperficie para definir a zona da FP.

Segundo Park et al. (1998), a estrutura vertical na ZA é caracterizada por uma camada superficial bem misturada e relativamente mais aquecida e menos salina composta pela ASA, que flui sobre a camada subjacente caracterizada por um mínimo de temperatura subsuperficial. Esta seria remanescente do inverno anterior que ficou tampada pelo aquecimento e dessalinização sazonal. A mistura dessas duas camadas de água forma a Água de Inverno. Segundo Pickard (1974), na ZA encontraremos a ASA ocupando uma fina camada de até aproximadamente $100 \mathrm{~m}$ de espessura centrada em $250 \mathrm{~m}$ de profundidade, com valores de temperatura variando entre $-1,9^{\circ} \mathrm{C}$ e $4^{\circ} \mathrm{C}$ (mínima de inverno e máxima de verão). Abaixo dessa camada flui a Água Profunda Circumpolar (APC, Orsi et al. 1995) com temperatura variando entre $1,5^{\circ} \mathrm{C}$ a $2,5^{\circ} \mathrm{C}$. A APC é centrada na coluna de água entre $300 \mathrm{~m}$ a $600 \mathrm{~m}$ e depois possui uma redução da temperatura para valores entre $0^{\circ} \mathrm{C}$ e $0,5^{\circ} \mathrm{C}$ em profundidades e até os $4000 \mathrm{~m}$ (Orsi et al. 1995).

$\mathrm{Na}$ ZSA, a água superficial tem uma maior amplitude nos valores de salinidade e temperatura. Esse fato pode ser explicado por uma maior influência das 
variações sazonais de aquecimento solar, chuva e evaporação. Nesta zona encontraremos a ASS nos primeiros $400 \mathrm{~m}$, com temperatura variando entre $4^{\circ} \mathrm{C} \mathrm{e}$ $14^{\circ} \mathrm{C}$ (mínima de inverno e máxima de verão). Abaixo desta massa d'água encontra-se a Água Intermediária Antártica (AIA). A AIA é a massa d'água predominante na ZSA. Essa camada é também caracterizada por um máximo de concentração de $\mathrm{O}_{2}$ dissolvido, definida nos limites termohalinos de $3^{\circ} \mathrm{C}$ a $6^{\circ} \mathrm{C}$ e salinidade em torno de 34,5 (Sverdrup et al. 1942, Silva et al. 2005).

Botnikov (1963) indica que a FP possui duas expressões (superficial e subsuperfícial) quanto à sua localização, sendo que estas não necessariamente coincidem. Moore et al. (1999) apontam que quando há separação dessas expressões, a parte mais subsuperficial tende a se localizar mais ao sul do que a superficial. Entretanto, Botnikov (1963) e More et al. (1999) indicam que a termodinâmica das massas d'água torna mais fácil para as águas menos densas da ZSA substituírem as águas mais densas da ASS. A definição da expressão da FP em subsuperfície seria a camada de mínima temperatura encontrada próxima a 200 m (Deacon 1937).

\section{MATERIAIS E MÉTODOS}

\section{AQUISIÇÃO DOS DADOS}

Um dos métodos mais antigos para medir a estrutura termal dos oceanos é através do lançamento de XBTs, que medem a temperatura da água do mar em função da profundidade da coluna d'água. O XBT é o instrumento mais barato, simples e de fácil manuseio entre todos os instrumentos disponíveis para medir a temperatura das camadas superiores do oceano, podendo ser lançado a partir de navios em movimento, ou mesmo a partir de aviões ou helicópteros (Thadathil et al. 2001).

O XBT é uma sonda descartável na forma de um mini-torpedo com um peso na ponta, munida de um termistor que é sensível à variação da temperatura da água em função da profundidade enquanto afunda. As medidas são transmitidas para um computador normalmente instalado a bordo de navio, através de um cabo muito fino de cobre que se rompe quando a profundidade máxima permitida ou desejada é atingida. Com um intervalo de tempo conhecido durante seu mergulho, a profundidade da sonda é deduzida a partir de uma equação de taxa de queda fornecida pelo fabricante onde para a faixa de profundidade entre $0 \mathrm{e}$ $800 \mathrm{~m}$ é observado um erro menor que $1 \mathrm{~m}$ (Thadathil et al. 2001). A equação de taxa de queda é representada na forma $Z(t)=A t-B t^{2}$, onde $Z$ é a profundidade da sonda, $t$ é o tempo decorrido desde o lançamento da sonda no mar, $A$ denota a velocidade terminal durante o mergulho do XBT e o termo quadrático fornece o somatório da perda de peso da sonda devido a movimentos horizontais durante a descida, que leva a uma redução da velocidade com o tempo (Gouretski et al., 2010). A partir da implementação do sistema MK-12 baseado em microcomputador, as medições podem ser visualizadas diretamente na tela do computador e registradas digitalmente em intervalos de profundidade de cerca de $0,6 \mathrm{~m}$ (Thadathil et al. 2001).

O número de estações em cada derrota do NApOc Ary Rongel na Passagem de Drake durante a OPXXII e OPXXIII, variou em função da disponibilidade de sondas para cada ano e da logística do navio, sendo 26 sondas XBT lançadas em 14 de novembro de 2003 (OPXXII) e 20 sondas XBT lançadas em 9 de novembro de 2004 (OPXXIII). As sondas foram lançadas entre as latitudes de $56^{\circ} \mathrm{S}$ e $61,4^{\circ} \mathrm{S}$ na OPXXII e entre $54,9^{\circ} \mathrm{S}$ e $60,5^{\circ} \mathrm{S}$ na OPXXIII, cobrindo um range de profundidades até no máximo $760 \mathrm{~m}$.

\section{ANÁLISE DOS DADOS DE XBT}

Após cada lançamento de XBT realizou-se uma análise preliminar de consistência dos dados através da analise da curva de temperatura em função da profundidade para cada perfil. Qualquer variação abrupta de temperatura, incoerente com os demais dados do perfil, foi identificada. Os dados considerados espúrios foram eliminados e após interpolados linearmente a partir dos valores de temperatura acima e abaixo da profundidade onde foram registrados. Além disso, os dados de cada perfil foram suavizados através da aplicação de uma janela móvel de $20 \mathrm{~m}$. Os primeiros valores registrados pelos XBTs $(0,6 \mathrm{~m}$ de profundidade) foram sempre descartados, pois apresentavam valores de temperatura superestimados como consequência do tempo de estabilização do sensor no momento da imersão na água logo após o lançamento. Assumimos, dessa forma, a profundidade de 1,3 m como a primeira a ser considerada na superfície. 
Como apontado por Gordon (1971), em função do mascaramento do gradiente vertical de temperatura na superfície do mar devido ao aquecimento das águas durante o verão austral, além de análises detalhadas dos dados de superfície, foi realizada uma análise particular da temperatura média da água do mar na profundidade de $100 \mathrm{~m}$, onde se encontra o mínimo de temperatura na coluna d'água na região de estudo.

Após a análise de consistência de todos os 46 perfis obtidos para os dois períodos estudados, extraiu-se a média de temperatura dos perfis de XBT para a ZA e ZSA nos meses de novembro de 2003 e 2004, assim como os respectivos desvios-padrão. Através da análise dos desvios padrão, assumimos que a região onde estes possuem maiores valores seja aquela de maior variabilidade das massas d'água da FP na ZA e ZSA.

Com base nos dados do perfil médio obtido anteriormente, elaborou-se também um gráfico das temperaturas médias da água do mar para a região de estudo durante a OPXXII e OPXXIII nas profundidades de 1,3 $\mathrm{m}$ (superfície), $50 \mathrm{~m}, 100 \mathrm{~m}$ e $200 \mathrm{~m}$. Esse gráfico auxilia a análise da estrutura termal da FP em função da profundidade ao longo das seções de derrota do navio em 2003 e 2004.

\section{CÁLCULO DOS GRADIENTES HORIZONTAIS DA TEMPERATURA DA SUPERFÍCIE DO MAR}

Para a definição da posição e da intensidade da FP a partir de dados de TSM obtidos pelos XBTs (1,3 $\mathrm{m})$, foram calculados os gradientes horizontais de TSM na direção norte-sul ao longo da derrota do NApOc Ary Rongel durante a OPXXII e a OPXXIII. Esses gradientes foram calculados através da seguinte equação:

$$
|\nabla T|=\sqrt{(\partial T / \partial x)^{2}+(\partial T / \partial y)^{2}}
$$

Onde $\nabla T$ é o gradiente horizontal de TSM, $\partial T / \partial x$ é a variação zonal de TSM e $\partial T / \partial y$ é a variação meridional de TSM.

O método indica a posição do maior valor do gradiente de TSM. Esse, por sua vez, aponta diretamente à latitude em que se localiza o maior contraste térmico tipicamente associado à posição da FP na região de estudo.

\section{CÁLCULO DA PROFUNDIDADE DA TERMOCLINA}

A termoclina é a região onde 0 gradiente vertical de temperatura da água do mar é máximo (Pickard 1974). Existem vários métodos utilizados para calcular a profundidade da termoclina. Nesse trabalho são utilizados dois métodos diferentes. 0 primeiro método consiste na aplicação direta do conceito do gradiente vertical de temperatura da água do mar $\left(\nabla_{z} T=\partial T / \partial z\right)$ e, dessa forma, determina-se a profundidade na qual ocorre a maior variação vertical da temperatura para cada perfil. $O$ segundo método é baseado no proposto por Kara et al. (2000). Assumindo-se que o topo da termoclina coincide com a base da camada isotermal (camada de mistura), tal método consiste na determinação da profundidade da base da camada isotermal.

O algoritmo de Kara et al. (2000) é implementado da seguinte maneira: primeiro é definida uma temperatura de referência $\left(T_{\text {ref }}\right)$ que é a TSM (1,3 m). Logo após, é procurada no perfil alguma região caracterizada por ter grande homogeneidade vertical de temperatura, ou seja,

$\left|T_{i}-T_{i-1}\right| \leq 0,1 \Delta T$

onde $\Delta T$ é a diferença de temperatura da água do mar entre a base da camada isotermal e o topo da termoclina (neste trabalho, o $\Delta T$ utilizado é de $0,5^{\circ} \mathrm{C}$ ). Caso esta região seja encontrada, a nova temperatura de referência passa a ser $T_{i}$. Caso contrário, continua sendo a TSM. O critério de seleção do $\Delta T$ foi baseado em testes feitos com outros valores (e.g. $\mathrm{T}=0,2^{\circ} \mathrm{C}, 0,5^{\circ} \mathrm{C}, 0,8^{\circ} \mathrm{C}$ e $1,0^{\circ} \mathrm{C}$ ), e o de $0,5^{\circ} \mathrm{C}$ foi escolhido por melhor representar o critério da diferença de temperatura.

Após a definição da temperatura de referência calculou-se a temperatura do topo da termoclina $\left(T_{b}\right)$ a partir de:

$$
T_{b}=\left\{\begin{array}{l}
T_{r e f}-\Delta T, \text { se } T_{i+1}<T_{i} \\
T_{r e f}+\Delta T, \text { se } T_{i+1}>T_{i}
\end{array}\right.
$$

Uma vez encontrada $T_{b}$ determina-se a profundidade equivalente $\left(Z_{b}\right)$. Caso $T_{b}$ esteja localizada entre o intervalo de profundidade $i$ e $i+1, Z_{b}$ é calculada 
através de interpolação linear. Assim, a profundidade da termoclina foi calculada para cada perfil obtido com os XBT na ZA e na ZSA individualmente. Posteriormente foram calculadas as médias destas profundidades considerando para cada OPERANTAR.

\section{RESULTADOS E DISCUSSÃO}

\section{OPERAÇÃO ANTÁRTICA XXII (2003)}

Durante a OPXXII, foram realizados 26 lançamentos de sondas XBT em uma seção linear que indica a derrota do NApOc Ary Rongel durante o dia 14 novembro de 2003. As sondas XBTs foram lançadas em uma malha de alta resolução onde a distância entre cada lançamento foi próxima a $0,25^{\circ}$ de latitude ou 15 milhas náuticas.

A Figura 1.a mostra o campo de TSM da região da Passagem de Drake no dia 14 de novembro de 2003. Os dados foram obtidos a partir do sensor AMSR-E (Advanced Microwave Scanning Radiometer do satélite Aqua) com uma resolução de $0,25^{\circ} \times 0,25^{\circ}$ lat/lon, sendo referentes a uma média de dados obtidos durante três dias. Além dos meandros da ACC e da fácil visualização da ZA e ZSA na FP (que esteve centrada próxima a 58 ㅇ na época em que os dados foram obtidos), é possível notar uma estrutura localizada próxima a $56,0^{\circ} \mathrm{S}-57,0^{\circ} \mathrm{S}$ e $53^{\circ} \mathrm{W}-55^{\circ} \mathrm{W}$ de forma elíptica, típica dos vórtices de mesoescala da região. Segundo Bryden (1983), é possível observar a presença de vórtices em diversas regiões do oceano Austral. Os processos de instabilidade baroclínica seriam a fonte principal de geração destes vórtices. Ainda, segundo o mesmo autor, a presença de vórtices proporciona um mecanismo eficiente para o transporte meridional de várias propriedades.

A estrutura termal da FP ao longo do transecto determinado pela posição de lançamento das sondas XBTs durante a OPXXII pode ser observada na Figura 2.a. Nota-se a existência de um gradiente de temperatura mais acentuado em profundidades mais rasas entre as latitudes de $57,5^{\circ} \mathrm{S}$ e $58^{\circ} \mathrm{S}$, indicando a região do núcleo da FP em novembro de 2003. É possível notar também que os meandramentos da CCA observados em superfície (Figura 1a), se refletem na coluna d'água onde, especialmente em profundidades menores que $200 \mathrm{~m}$, observam-se núcleos com temperaturas mais baixas $\left(\sim 0,5^{\circ} \mathrm{C}\right)$ do que as águas adjacentes da ZSA $\left(\sim 4^{\circ} \mathrm{C}\right)$ na FP. Esses núcleos se refletem na profundidade da termoclina (denotada aqui pelos máximos gradientes verticais termais) e são, possivelmente, o sinal da presença de feições de mesoescala como vórtices ou meandros da CCA. O gradiente termal entre as águas das duas zonas compreendidas pela FP na latitude próxima de $58 \stackrel{\circ}{S}$ se estende até a profundidade máxima de amostragem das sondas XBTs em $760 \mathrm{~m}$. a)

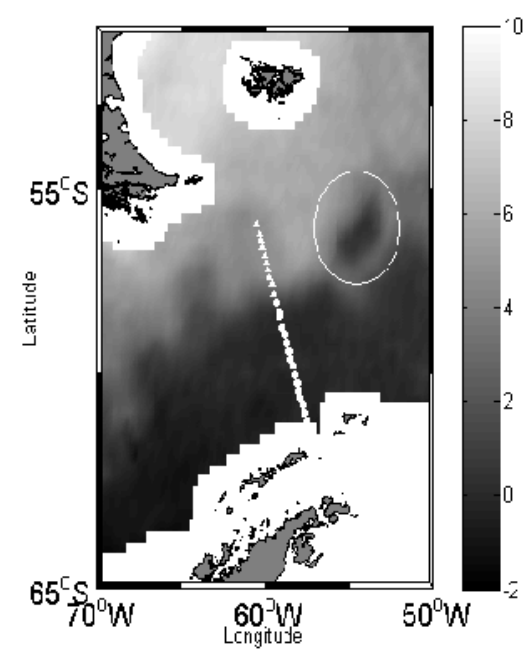

b)

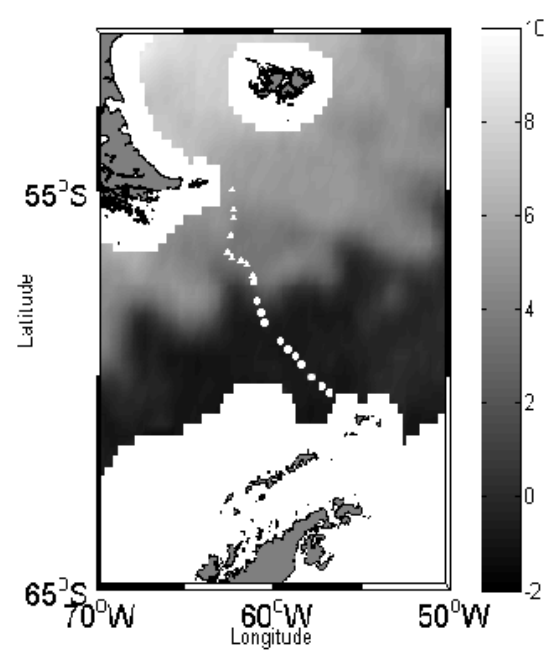

c)

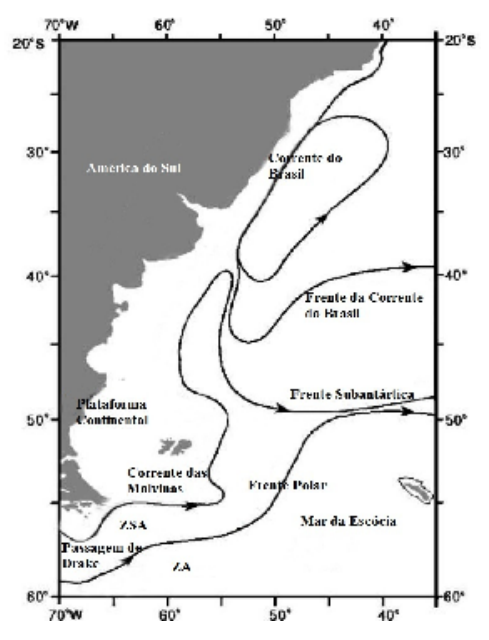

FIGURA 1 - a) Mapa de TSM gerado a partir de dados AMSR-E do dia 14 de novembro de 2003 (OPXXII). Os triângulos e círculos brancos indicam os locais de lançamento de XBTs sobre a ZSA e ZA, respectivamente. A elipse branca indica a posição de um vórtice de mesoescala originado na ZA que migrou para a ZSA. b) Mapa de TSM gerado a partir de dados AMSR-E do dia 9 de novembro de 2004 (OPXXIII). Os triângulos e círculos brancos indicam os locais de lançamento de XBTs sobre a ZSA e ZA, respectivamente. c) Região do oceano Atlântico Sudoeste, Passagem de Drake e setor Atlântico do oceano Austral com as principais frentes oceanográficas e correntes marinhas associadas 
Os perfis médios de temperatura da água do mar obtidos na ZA e ZSA durante a OPXXII podem ser observados na Figura 3.a. Para a ZA é possível perceber, analisando o padrão da curva em função da profundidade, que o mínimo de temperatura se encontra a $80 \mathrm{~m}$ com uma temperatura próxima a $0,4^{\circ} \mathrm{C}$. O perfil médio de temperatura das águas da ZA em superfície apresenta um valor de $-0,2^{\circ} \mathrm{C} \pm 0,4$.
Esse desvio padrão representa uma medida da magnitude do espalhamento ou dispersão dos dados em relação à temperatura média da superfície do mar. A região da ZSA apresentou uma média de temperatura da água do mar em superfície de $4,4^{\circ} \mathrm{C} \pm$ 0,4 . O maior desvio padrão para a ZSA $\left(0,5^{\circ} \mathrm{C}\right)$ ocorreu próximo a $60 \mathrm{~m}$.

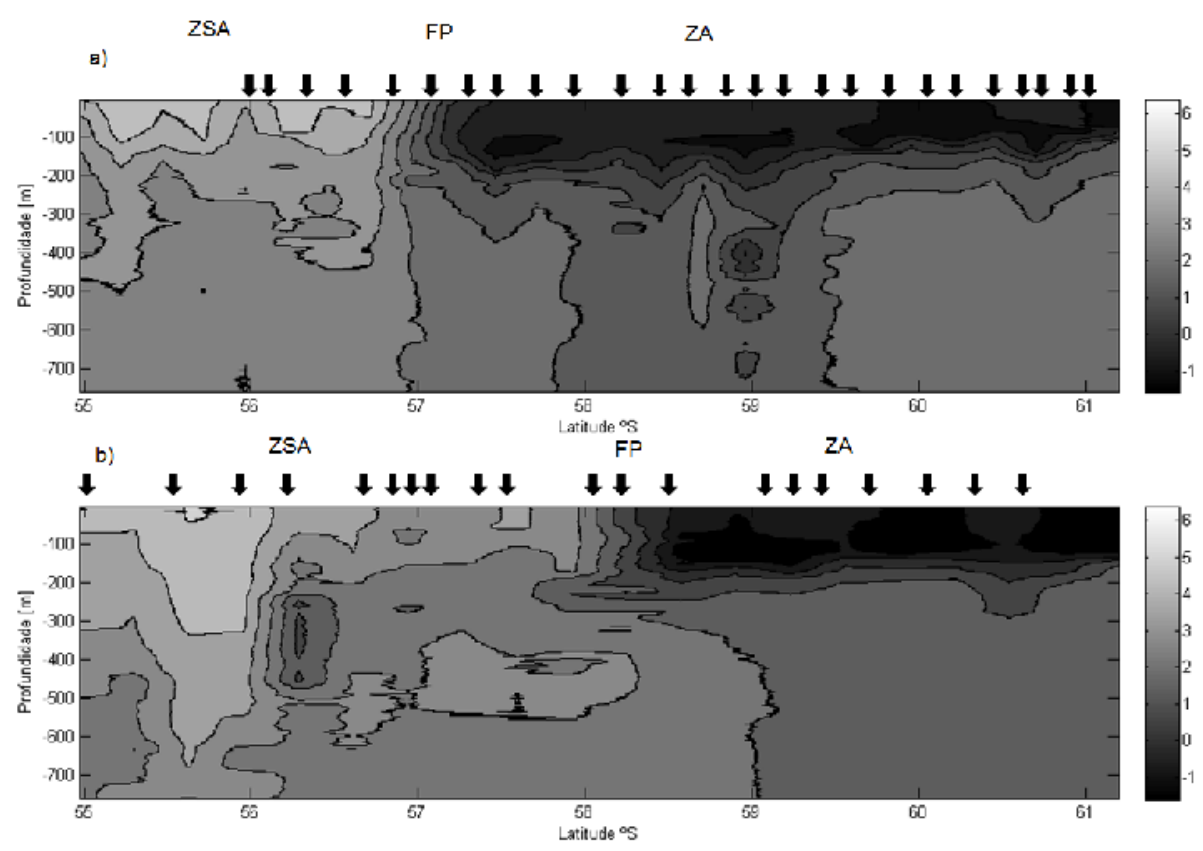

FIGURA 2 - Seções de temperatura da água do mar em alta resolução entre as profundidades de $1.3 \mathrm{~m}$ e $760 \mathrm{~m}$ obtida a partir dos dados de XBTs ao longo da derrota do NApOc Ary Rongel durante a OPXXII (a) e OPXXIII (b). Incluem-se nessas seções a localização da Frente Polar (FP), da Zona Subantártica (ZSA) e da Zona Antártica (ZA). As setas negras indicam a posição de lançamento das sondas XBTs.

\section{OPERAÇÃO ANTÁRTICA XXIII (2004)}

20 lançamentos de XBT foram realizados na OPXXIII. Ao contrário da OPXXII, é possível perceber que o navio realizou um trajeto menos retilíneo dessa vez, o que ocorreu por consequência do mau tempo característico da região da Passagem de Drake. No mapa de TSM referente ao período estudado, podese observar que os meandros da CCA estão bem evidentes na época do cruzeiro em novembro de 2004 (Figura 1b). São também observadas estruturas de mesoescala com formas elípticas ou arredondadas, típicas dos vórtices que são comuns aos dois lados da FP.

Nota-se uma forte subdução de águas de origem polar entre $58^{\circ} \mathrm{S}$ e $56^{\circ} \mathrm{S}$, associado a uma intensa formação de estruturas instáveis típicas do processo de interleaving (Figura $2 \mathrm{~b}$ ) onde águas de diferentes temperaturas e salinidades permanecem em contato nos oceanos, sendo assim, características de uma região de relativo alto gradiente lateral de temperatura e salinidade (neste caso, uma frente oceânica). Centrado em aproximadamente $55^{\circ} \mathrm{S}$, um forte rebaixamento da profundidade da termoclina é observado, sendo esse provavelmente associado a um grande vórtice de núcleo quente da região da ZSA. Na ZA, a profundidade típica da termoclina foi de aproximadamente $200 \mathrm{~m}$, com águas superficiais relativamente mais frias que as águas localizadas na camada logo abaixo.

A temperatura média registrada em superfície para a ZSA foi de $3,8^{\circ} \mathrm{C} \pm 0,8$ (Figura $3 b$ ). $O$ maior desvio padrão do perfil médio dessa região foi de $1,1^{\circ} \mathrm{C}$ a $240 \mathrm{~m}$. Na ZA, a temperatura média da água do mar na superfície foi de $-0,6^{\circ} \mathrm{C} \pm 0,6$. $O$ maior 
desvio padrão ocorreu próximo a $80 \mathrm{~m}$, com um valor de $0,8^{\circ} \mathrm{C}$.

\section{ZONAS DELIMITADAS DA FRENTE POLAR}

O perfil médio de temperatura na ZA e na ZSA obtido com os dados das duas operações antárticas está apresentado na Figura 3c. Assim como observado em outros trabalhos (Orsi et al. 1995, Thompson et al. 2005), o contraste entre a ZA e a ZSA é bem evidente. As características oceanográficas gerais da região de estudo podem, então, ser resumidas dessa forma:

- Em profundidades maiores que $200 \mathrm{~m}$, a ZA apresenta águas com temperatura inferior a $2^{\circ} \mathrm{C}$ e cerca de $2,5^{\circ} \mathrm{C}$ mais quentes que as águas superficiais;

- Em profundidades maiores que $200 \mathrm{~m}$, a ZSA apresenta águas com temperatura entre $2,5 \mathrm{e}$ $3^{\circ} \mathrm{C}$ e cerca de 1 a $1,5^{\circ} \mathrm{C}$ mais frias que as águas superficiais;

- A ZA apresenta uma camada de mistura mais estável até $100 \mathrm{~m}$ de profundidade, onde é localizado o topo da termoclina;
- A ZSA apresenta uma termoclina que se estende desde a superfície até a profundidade de $200 \mathrm{~m}$.

A ZA apresentou temperatura média superficial de $-0,4^{\circ} \mathrm{C} \pm 0,3$. Na ZSA, o valor da temperatura média superficial foi de $4,1^{\circ} \mathrm{C} \pm 0,4$. Os resultados encontrados concordam com o que já foi descrito no trabalho de Bagriantsev et al. (1989). Na ZA a estratificação ocorre de maneira que a ASA, relativamente mais fria $e$ menos salina por conseqüência do derretimento do gelo marinho, sobrepõe-se às águas mais quentes e menos salinas da APC. É possível observar na Figura 3c um forte gradiente termal vertical na interface entre as duas massas de água. Ainda na ZA, a temperatura mínima de $-0,6^{\circ} \mathrm{C}$ ocorre próxima a $100 \mathrm{~m}$ de profundidade. $\mathrm{A}$ partir desta profundidade, a temperatura da água aumenta e tende a valores próximos de $2^{\circ} \mathrm{C}$ que são característicos para ZA nesta região para essa profundidade, assim como apresentados por Orsi et al . (1995).
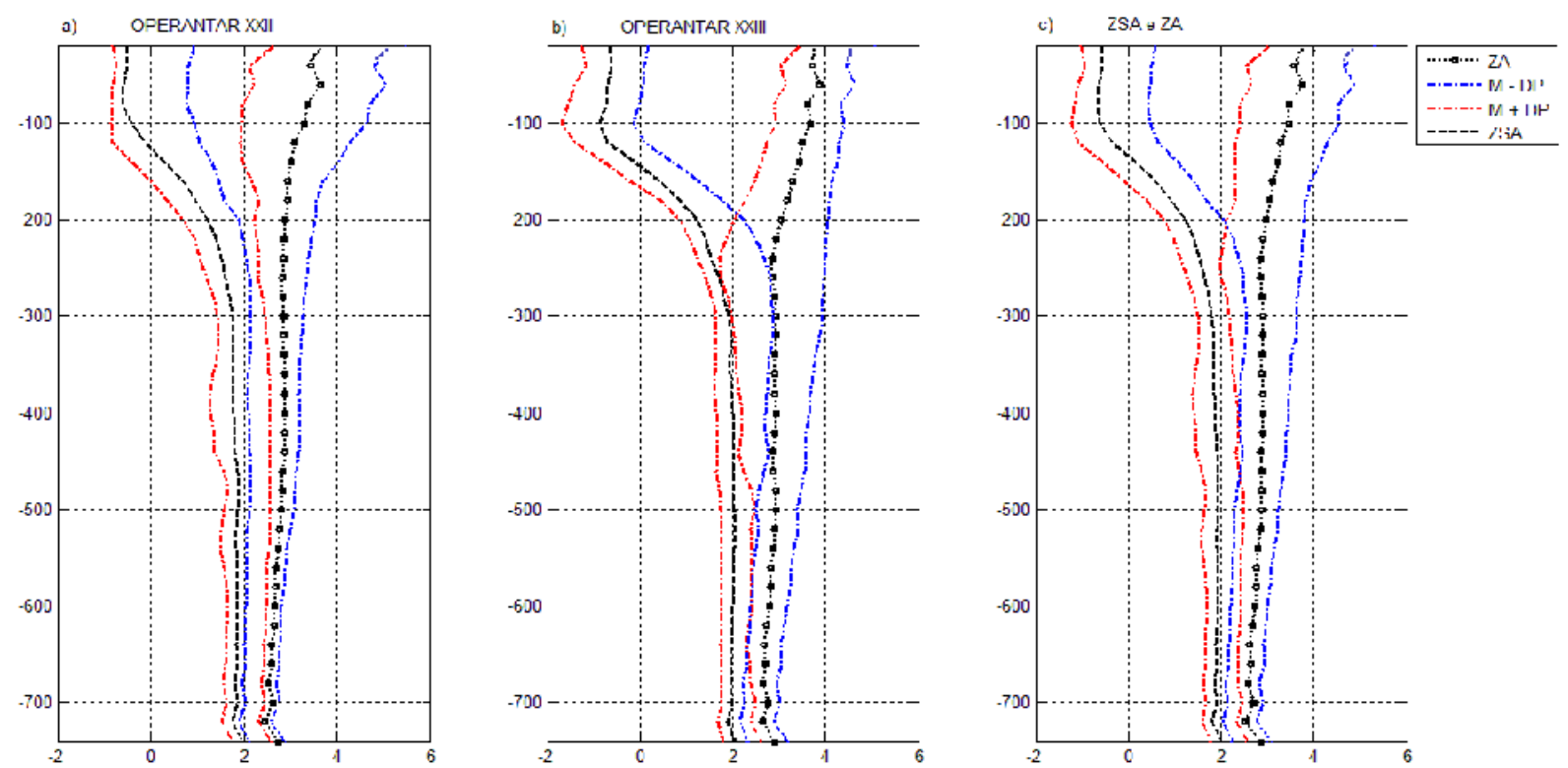

FIGURA 3 - Comparação dos perfis médios verticais de temperatura da água do mar na região da Frente Polar obtidos durante as OPERANTAR XXII e XXIII através de sondas XBTs. a) Perfil médio da ZA e da ZSA com a temperatura média mais o desvio padrão (M+DP) e com a média menos o desvio padrão (M-DP) durante a OPERANTAR XXII. b) Perfil médio da ZA e da ZSA com a temperatura média mais o desvio padrão (M+DP) e coma média menos o desvio padrão (M-DP) durante a OPERANTAR XXIII. c) Perfil médio da ZA e da ZSA com a temperatura média mais o desvio padrão (M+DP) e com a média menos o desvio padrão (M-DP) com dados obtidos durante as OPERANTAR XXII e XXXIII juntas. 
$\mathrm{Na} Z S A$, ao contrário da ZA, não se percebe uma variação tão abrupta na temperatura da água do mar a partir dos $100 \mathrm{~m}$ de profundidade. A redução da temperatura com a profundidade ocorre de maneira mais suave e desde a superfície, pois a camada de mistura é menos evidente. Em profundidades, a partir de $200 \mathrm{~m}$, a temperatura também apresenta aparente estabilidade com valores próximos a $2,5^{\circ} \mathrm{C}$ na coluna d'água até a profundidade máxima amostrada pelos XBTs.

\section{POSIÇÃO DA FRENTE POLAR}

Observando-se as imagens de TSM e os perfis de alta resolução obtidos para as regiões analisadas nesse trabalho (Figura 1 e 2), é possível apontar como posição média da FP as latitudes entre $57^{\circ} \mathrm{S}$ e $58^{\circ} \mathrm{S}$ na região de estudo deste trabalho. Thompson et al. (2005), com base no trabalho desenvolvido por Orsi et al. (1995), determinaram a latitude de $58,4^{\circ} \mathrm{S}$ como a posição média da FP. Entretanto, os autores relatam também que a posição instantânea da FP pode variar por centenas de quilômetros, dependendo da longitude em que a frente se situa ao redor do continente antártico.

Na Figura 4 são apresentadas as temperatura da água do mar registradas nos dados originais dos XBTs em quatro profundidades diferentes $(1,3 \mathrm{~m}, 50$ $\mathrm{m}, 100 \mathrm{~m}$ e $200 \mathrm{~m}$ ) ao longo das latitudes amostradas pelo navio. A Figura também apresenta os gradientes horizontais de temperatura da água do mar encontrados ao longo das diversas latitudes para cada uma das quatro profundidades em questão. Durante a OPXXII, houve concordância quanto à posição em latitude do maior gradiente horizontal termal em todas as profundidades analisadas. Como evidenciado anteriormente a partir das imagens de satélite e dos perfis de alta resolução, 0 maior gradiente horizontal termal da região da FP se situou em cerca de $57,7^{\circ} \mathrm{S}$ de latitude para todas as profundidades.

A OPXXIII foi a que apresentou maior variação latitudinal da FP. Em superfície e a $50 \mathrm{~m}$ o maior gradiente horizontal foi localizado a $57,5^{\circ} \mathrm{S}$. A $100 \mathrm{~m}$ de profundidade, a latitude onde se encontrava a FP era $58,1^{\circ} \mathrm{S}$, enquanto que a $200 \mathrm{~m}$ a latitude encontrada foi de $57,3^{\circ} \mathrm{S}$. Essa variação pode ter sido ocasionada por uma condição sinótica (como, por exemplo, a passagem de uma frente fria com ventos intensos), pela derrota do navio não ter ocorrido de forma perpendicular aos gradientes de temperatura da região ou pela dinâmica de mesoescala ocasionada pelo subdução de água antártica e interleavings já descritos. Os valores da posição latitudinal da FP em superfície e subsuperfície são compatíveis com resultados obtidos por outros autores. A Tabela 1 compara os resultados obtidos aqui para a posição em latitude da FP a partir de dados de gradientes horizontais termais para a superfície e subsuperfície com aqueles descritos por Read et al. (1995), Ikeda et al. (1989), Orsi et al. (1995) e Sprintall et al. (2003). Nesse trabalho, a posição média da FP coincidiu com os autores citados acima no que se refere à comparação entre as expressões de superfície e de subsuperfície com exceção dos obtidos por Sprintall et al. (2003) que podem estar relacionados a metodologia aplicada, pois este considera a médias entre as profundidades 100, 200 e $300 \mathrm{~m}$. Durante a OPXXII, de novo houve diferença entre os valores encontrados por Sprintall et al. (2003), porém para o restante dos resultados,não houve diferença entre as duas expressões, entretanto, a FP apresentou-se mais ao norte em subsuperfície do que em superfície durante a OPXXIII.

Para a OPXXII e nas três profundidades mais rasas que $200 \mathrm{~m}$, os gradientes latitudinais de temperatura da água do mar foram mais altos e com valores entre $0,07^{\circ} \mathrm{C} . \mathrm{km}^{-1}$ a $0,09^{\circ} \mathrm{C} . \mathrm{km}^{-1}$. Em $200 \mathrm{~m}$, o gradiente encontrado foi de $0,03^{\circ} \mathrm{C} \cdot \mathrm{km}^{-1}$. Observandose a Figura 4a e 4b, a queda acentuada de temperatura e o pico do máximo gradiente na transição entre as duas zonas da FP ficam claramente evidenciados. Assim como durante a OPXXII, a profundidade de $200 \mathrm{~m}$ durante a OPXXIII também apresentou menor gradiente horizontal termal em comparação com aquele das demais profundidades, com um valor de $0,02^{\circ} \mathrm{C} \cdot \mathrm{km}^{-1}$. Para as três primeiras profundidades durante a OPXXIII, o valor do gradiente encontrado variou entre $0,04^{\circ} \mathrm{C} . \mathrm{km}^{-1}$ e $0,05^{\circ} \mathrm{C} . \mathrm{km}^{-1}$. 
TABELA 1 - Comparação da posição latitudinal da FP na Passagem de Drake obtida nos demais trabalhos com ênfase nas expressões superficiais e subsuperficiais. '-' indica que não havia informação disponível.

\begin{tabular}{lll}
\hline Referência & Subsuperfície $\left({ }^{\circ} \mathbf{S}\right)$ & Superfície $\left({ }^{\circ} \mathbf{S}\right)$ \\
Sprintall et al. (2003) & $58-59^{\star *}$ & $58-59^{\star *}$ \\
Orsi et. al. (1995) & - & 58,4 \\
Read et al. (1995) & 57,8 & 58,2 \\
Ikeda et al. (1989) & 56,4 & - \\
OPERANTAR XXII & $57,7^{\star}$ & 57,7 \\
OPERANTAR XXIII & $57,5^{*}$ & 58,1 \\
\hline
\end{tabular}

*média entre as profundidades de 50, 100 e $200 \mathrm{~m}$.

** média entre as latitudes apresentadas.

A posição média da FP durante a OPXXII e a OPXXIII esteve muito próxima a descrita em Orsi et al. (1995), que é de $58,4^{\circ} \mathrm{S}$. Porém encontrou-se mais a sul do que reportado por Sprintall et al. (2003). Estas diferenças podem ser associadas a variabilidades na circulação, posição e transporte da CCA relacionados a alterações dos padrões de circulação atmosférica como proposto por Sokolov \& Rintoul (2009b) causados por mudanças na distribuição dos campos de pressão atmosférica (Lovenduski \& Gruber, 2005). Na Figuras 4a e 4c está muito evidente a abrupta queda de temperatura ao sul da latitude de $57^{\circ} \mathrm{S}$. Concordando também com o que foi apontado por Moore et al. (1999), a posição média da FP apresentou grande similaridade com aquelas definidas em outros estudos (Gille 1994, Orsi et
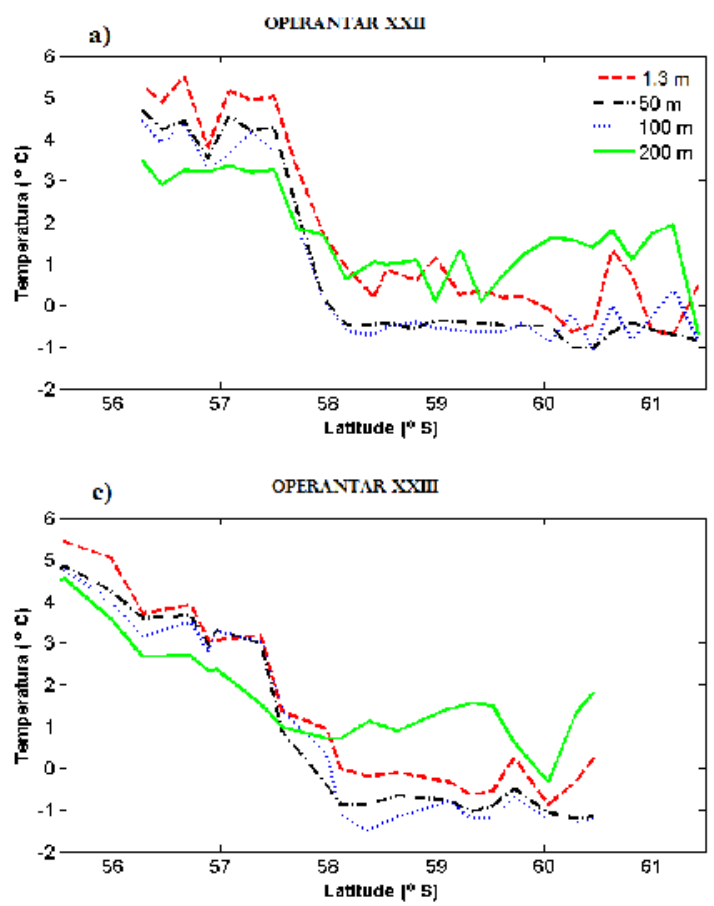

al.1995, Belkin e Gordon 1996). Esses trabalhos também foram realizados considerando a expressão da FP em subsuperfície. Lutjeharms \& Valentine (1984) indicam que as expressões de subsuperfície da FP estão situadas predominantemente mais ao norte que a expressão em superfície.

Sprintall (2003) encontrou a localização média da FP na Passagem de Drake mais ao norte do que as suas posições históricas registrada por Orsi et al. (1995). Moore et al. (1999) indicam que, apesar da localização das expressões de superfície e subsuperfície da FP poderem ser diferentes em períodos curtos (ou em escalas de espaço pequenas), a sua localização média está intimamente associada a ambas camadas em grande parte do oceano Austral.
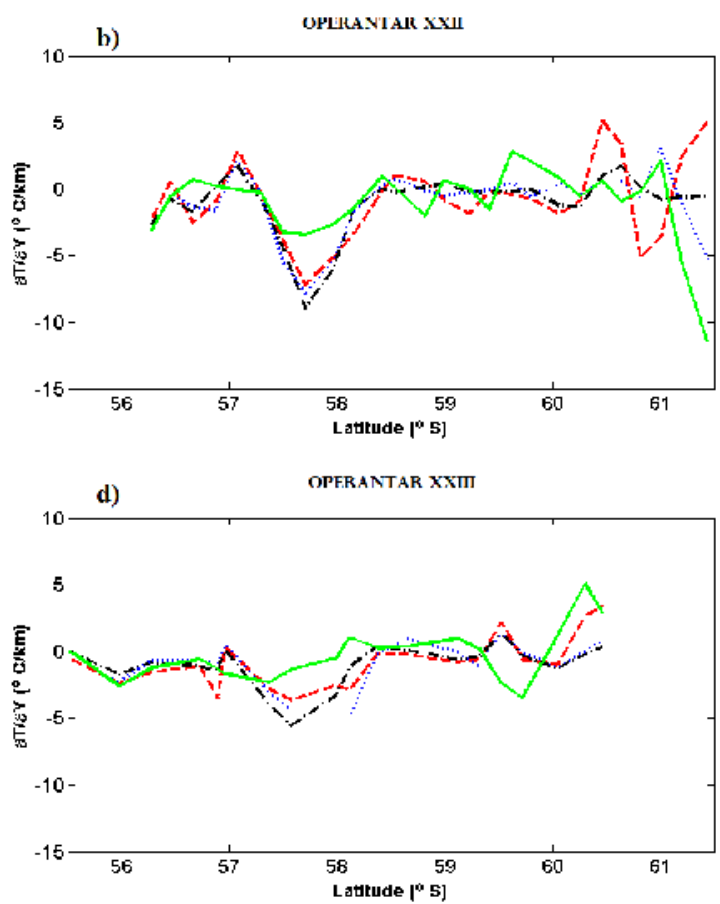

FIGURA 4 - Perfis dos valores de temperatura em 4 profundidades $(1,3 \mathrm{~m}, 50 \mathrm{~m}, 100 \mathrm{~m}$ e $200 \mathrm{~m})$ em função da latitude calculados para a OPERANTAR XXII e OPERANTAR XXIII (painéis da esquerda) e de gradiente de temperatura nas mesmas 4 profundidades também em função da latitude (painéis da direita). 


\section{PROFUNDIDADE DA TERMOCLINA}

A importância da determinação da profundidade da termoclina se encontra na possibilidade de descrever mais realisticamente processos de interação oceano-atmosfera locais que impactam em regimes de circulação globais. Esta informação pode servir para melhorar as parametrizações utilizadas em modelos de circulação oceânica e atmosférica melhorando as previsões de tempo e clima. Os resultados provenientes da aplicação dos dois métodos descritos na Seção 2.2 estão apresentados na Tabela 2. Os resultados foram obtidos utilizando os dados de temperatura da água do mar média calculados para as duas operações antárticas tratadas aqui. É possível observar diferenças nas profundidades da termoclina obtida com o método desenvolvido por Kara et al. (2000) e os resultados obtidos pelo método do gradiente.

É importante considerar que o método descrito por Kara et al. (2000) faz uso de interpolação linear para obter a profundidade do topo da termoclina, enquanto que o método do gradiente simplesmente aponta a região (profundidade) onde a temperatura estaria variando mais em função da profundidade $(\Delta \mathrm{z})$.

As diferenças encontradas entre os valores da profundidade da termoclina podem ser explicadas pela diferença nessas metodologias. Não necessariamente a região do topo da termoclina obtida pelo primeiro método vai coincidir com a região de maior gradiente vertical de temperatura na coluna d'água obtido com o segundo.

TABELA 2 - Profundidade da termoclina obtida para a OPXXII e OPXXIII através do método de Kara et al. (2000) e do gradiente vertical. ZSA=Zona Subantártica; $Z A=$ Zona Antártica. Os valores entre parênteses nas duas ultimas colunas indicam à profundidade $(\mathrm{m})$ onde o maior gradiente foi encontrado.

\begin{tabular}{lllll}
\hline Operação Antártica & \multicolumn{1}{l}{ Profundidade da Termoclina $(\mathbf{m})$} & \multicolumn{2}{l}{ Gradiente vertical de temperatura $\left({ }^{\circ} \mathbf{C} \mathbf{~}^{-1}\right)$} \\
\hline & ZSA & ZA & ZSA & ZA \\
\hline XXII & 102 & 118 & $0,007(101 \mathrm{~m})$ & $0,02(141 \mathrm{~m})$ \\
XXIII & 174 & 116 & $0,007(180 \mathrm{~m})$ & $0,03(141 \mathrm{~m})$ \\
\hline
\end{tabular}

\section{CONCLUSÃO}

Esse trabalho realizou um estudo da estrutura termal da região da FP durante os meses consecutivos de novembro entre os anos de 2003 e 2004. Durante esses períodos, o GOAL, realizou uma amostragem em alta resolução da temperatura da água do mar em função da profundidade ao longo da Passagem de Drake cruzando a FP.

Os perfis de temperatura média ao longo das profundidades de $1,3 \mathrm{~m}, 50 \mathrm{~m}, 100 \mathrm{~m}$ e $200 \mathrm{~m}$ mostram que a diferença de temperatura entre a ZA e ZSA ao longo da FP é de cerca de $4,5{ }^{\circ} \mathrm{C}$ na superfície, enquanto que em subsuperfície é de $4,2^{\circ} \mathrm{C}$. Além disso, os perfis mostram que a ZA apresenta um maior gradiente vertical na região da termoclina em relação à ZSA. Estudos anteriores (Lutjeharms \& Valentine 1984, Dong et al. 2006) sugerem que a expressão de superfície da FP, em geral, se localiza mais ao sul do que a de subsuperfície. Embora durante a OPXXII a posição da frente estivesse quase que uniformemente distribuída ao longo da profundidade, durante a OPXXIII a FP ficou situada a $1^{\circ}$ de latitude mais ao sul em sua expressão subsuperficial em relação a superficial. Essa diferença pode ser justificada pela variabilidade de alta freqüência associada à mesoescala ou a processos turbulentos típicos da região de estudo. É importante mencionar que a diferença entre as expressões de superfície e subsuperfície na posição da FP podem ocorrer em períodos relativamente curtos. Na média, entretanto, a localização de ambas está intimamente associada (Moore et al.1999).

Foram utilizados dois métodos para a determinação da profundidade da termoclina, um baseado na determinação da profundidade do topo da camada estável ou de mistura (Kara et al. 2000) e o outro com base no gradiente vertical da temperatura da 
água do mar. Apesar da diferença nos resultados obtidos através das duas metodologias, foi possível perceber uma coerência quando se analisaram as duas zonas da FP durante os dois anos estudados. Durante a OPXXII, a termoclina esteve mais rasa na ZSA em relação à OPXXIII. Essa diferença pode ter sido ocasionada pela variabilidade de mesoescala dessa região, o que faz com que a termoclina oscile em função da presença ou ausência de vórtices quentes (ficando mais funda) ou frios (ficando mais rasa). É possível observar uma certa coerência nos valores obtidos com as diferentes metodologias, com a profundidade da termoclina oscilando entre 100 e $180 \mathrm{~m}$.

Apesar dos relativamente poucos dados obtidos pelo GOAL na região de estudo durante os meses de novembro dos anos 2003 e 2004, estes coincidiram com aqueles descritos na literatura no que diz respeito à estrutura termal das duas zonas que formam a FP. É importante realizar estudos descritivos como o apresentado aqui, que podem incrementar nosso conhecimento sobre a natureza da FP e a variabilidade interanual do seu posicionamento. A modelagem numérica de circulação oceânica pode ser altamente beneficiada pela disponibilidade de dados de temperatura da água do mar e da profundidade da termoclina. A determinação precisa da espessura da camada de mistura oceânica na região da Passagem de Drake é importante para auxiliar o entendimento do acoplamento oceano-atmosfera e das teleconexões entre o oceano Austral e o oceano Atlântico Sul.

\section{AGRADECIMENTOS}

Esse trabalho foi financiado pelos projetos GOAL (550370/2002-1), INTERCONF (557284/2005-8) e SOS-CLIMATE (520189/2006-0) com o apoio do CNPq e PROANTAR. O estudo também é uma contribuição ao INCT da Criosfera. Os batitermógrafos descartáveis foram gentilmente cedidos pela Marinha do Brasil e AOML/NOAA (Atlantic Oceanographic and Meteorological Laboratory / National Oceanic and Atmospheric Administration). Os autores agradecem o comandante e tripulação do NApOc Ary Rongel pela inestimável ajuda durante o trabalho de campo in situ. Também agradecemos a Comissão Interministerial para os Recursos do Mar (CIRM), O Ministério do Meio Ambiente, dos Recursos Hídricos e da Amazônia Legal (MMA) e o Ministério da Ciência, Tecnologia e Inovação (MCTI) pelo suporte às atividades do GOAL. MM Mata agradece o apoio recebido do CNPq (bolsa PQ-2 307630/2009-9).

\section{REFERÊNCIAS}

BAGRIANTSEV, N.V., GORDON, A.L. \& HUBER, B.A. 1989. Weddell Gyre: temperature maximum stratum. Journal of Geophysical Research, 94: 8331-8334.

BELKIN, I. M., \& A. L. GORDON, 1996. Southern Ocean fronts from the Greenwich meridian to Tasmania. Journal of Geophysical Research, 101:3675-3696.

BOTNIKOV, V.N. 1963. Geographic position of the Antarctic convergence zone in the Southern Ocean (English translation). Soviet Antarctic Expedition Information Bulletin.,4: 324-327.

BROWN, J. 1998. Ocean Circulation. England: The Open University, v.1.

BRYDEN, H.L. 1983. The Southern Ocean. Pp.265-277. In: ROBINSON, A.R. (ed.). Eddies in Marine Science. SpringerVerlag Berlin Heidelberg. 609p.

CUNNINGHAM, S. A., ALDERSON, S. B., KING, B. A., BRANDON, M.A. 2003. Transport and variability of the Antarctic Circumpolar Current in Drake Passage. Journal of Geophysical Research, 108:1-17.

DEACON, G.E.R. 1937. The hydrology of the Southern Ocean. Discovery Reports, 15:1-124.

DONG S., SPRINTALL J., \& GILLE S.T. 2006 Location of the Antarctic Polar Front from AMSR-E Satellite Sea Surface Temperature Measurements. American Meteorological Society, 36: 2075-2089.

GILLE, S.T. 1994. Mean sea surface height of the Antarctic Circumpolar Current from Geosat data: Method and application, Journal of. Geophysical Research, 99:18,255-18,273.

GORDON A.L. 1967. Structure of Antarctic waters between $20^{\circ} \mathrm{W}$ and $170^{\circ}$ W. In. Antarctic map folio series, Folio 6, Bushnell V.C. (Ed.). American Geography Society, 100p.

GORDON, A.L. 1971. Antarctic polar front zone. Pp. 205-221.In: Reid, J.L. (Ed.). Antarctic Oceanology I, Antarctic Res. Ser. Vol. 15. Amer. Geophys. Union.

GOURETSKI, V.V., \& DANILOV, A.I. 1993. Weddell Gyre: Structure of the eastern boundary, Deep Sea Research,40:561-582.

GOURETSKI, V. \& ROSEGHETTI, F. 2010. On depth and temperature biases in bathythermograph data: Development of a new correction scheme based on analysis of a global ocean database, Deep Sea Research, 57:812-833.

GLORIOSO P.D., PIOLA, A.R \& LEBEN, R.R. 2005. Mesoscale eddies in the Subantarctic Front - Southwest Atlantic. Scentia Marina, 69: 7-15.

HEYWOOD, K. \& KING, B. 2002. Water masses and baroclinic transports in the South Atlantic and Southern oceans. Journal of Marine Research, 60:639-676.

IKEDA, Y., SIEDLER, G. \& ZWIERZ, M. 1989. On the variability of Southern Ocean front locations between Southern Brazil and the Antarctic Peninsula, Journal of Geophysical Research, 94: 4757-4762.

KARA, A.B., ROCHFORD, P.A. \& HULBURT, H.E. 2000. An optimal definition for ocean mixed layer depth, Journal of Geophysical Research.,105(C7):16,803-16,821.

LOVENDUSKI, N. S., GRUBER, N. 2005. Impact of the Southern Annular Mode on Southern Ocean circulation and biology. Geophysical Research Letters, 32:1-4.

LUTJEHARMS, J.R.E. \& VALENTINE H.R. 1984. Southern ocean thermal fronts south of Africa, Deep Sea Research Part $A$ : 
Oceanographic Research Papers, 31. Issue 12. p.1461-1475, ISSN 0198-0149, DOI: 10.1016/0198-0149(84)90082-7.

MOORE, J.K., ABBOTT, M.R., \& RICHMAN J.G. 1997. Variability in the location of the Antarctic Polar Front $\left(90^{\circ}-20^{\circ} \mathrm{W}\right)$ from satellite sea surface temperature data, Journal of Geophysical Research, 102: 27,825-27,833.

MOORE, J.K., ABBOTT, M.R., \& RICHMAN J.G. 1999. Location and dynamics of the Antarctic polar front from satellite sea surface temperature data. Journal of Geophysical Research, 104: 3059-3073.

PARK, Y.H., CHARRIAUD, E., \& FIEUX M. 1998. Thermohaline structure of the Antarctic Surface WaterrWinter Water in the Indian sector of the Southern Ocean. Journal of Marine Systems, 17: 5-23.

PEZZI, L. P., SOUZA, R. B., DOURADO, M. S., MATA, M.M., GARCIA, C.A.E. \& SILVA-DIAS, M.A.F. 2005. Oceanatmosphere in situ observations at the Brazil-Malvinas Confluence region. Geophysical Research Letters, 32, doi:10.1029/2005GL023866

PEZZI, L.P., SOUZA, R.B., ACEVEDO, O., WAINER, I., MATA, M.M., GARCIA, C.A.E. \& CAMARGO, R. 2009 Multiyear measurements of the oceanic and atmospheric boundary layers at the Brazil-Malvinas Confluence Region Journal of Geophysical Research, $114 . \quad$ D19103. doi:10.1029/2008JD011379

PICKARD, G. L. 1974. Oceanografia Física Descritiva, $2^{\circ}$ ed.: Fundação de Estudos do Mar, Rio de Janeiro, RJ, 180p.

ORSI, A.H., WHITWORTH III, T. \& NOWLIN Jr, W.D. 1995. On the meridional extent and fronts of the Antarctic Circumpolar Current. Deep-Sea Research I, 42: 641-673.

READ, J.F., POLLARD, R.T., MORRISON, A.I., \& SYMON, C. 1995. On the southerly extent of the Antarctic Circumpolar
Current in the southeast Pacific, Deep Sea Research, Part II, 42: 933-954..

SPRINTALL, J. 2003. Seasonal to interannual upper-ocean variability in the Drake Passage. Journal of Marine Research, 61: 27-57.

SILVA, A. C., ARAÚJO, M. \& BOURLÈS, B. 2005. Variação sazonal da estrutura de massas de água na plataforma continental do Amazonas e área oceânica adjacente. Revista Brasileira de Geofísica, 23(2):145-157.

SVERDRUP, H.U., JOHNSON, M.W. \& FLEMING, R.H. 1942. The Oceans. Prentice-Hall, 1085p.

SOKOLOV, S. \& RINTOUL, S. 2009. Circumpolar structure and distribution of the Antarctic Circumpolar Current fronts: 1. Mean circumpolar paths. Journal of Geophysical Research, 114:1-19.

SOKOLOV, S. \& RINTOUL, S. 2009b. Circumpolar structure and distribution of the Antarctic Circumpolar Current fronts: 2. Variability and relationship to sea surface height. Journal of Geophysical Research, 114:1-15

THADATHIL, P., GHOSH, A.K., SARUPRIA, J.S. \& GOPALAKRISHNA, V.V. 2001. An interactive graphical system for XBT data quality control and visualization. Computers \& Geosciences, 27: 867-876.

THOMPSON, A.F., GILLE, S.T., MACKINNON, J.A. \& SPRINTALL, J. 2005. Spatial and temporal patterns of small-scale mixing in Drake Passage. Journal of Physical Oceanography, 36: 572592.

WHITWORTH III, T. \& NOWLIN, W. 1987. Water Masses and Currents at the Greenwich of the Southern Ocean at the Greenwich Meridian. Journal of Geophysical Research, 92: 6462-6476.

Submetido - 09/12/2011

Aceito - 11/06/2012 
